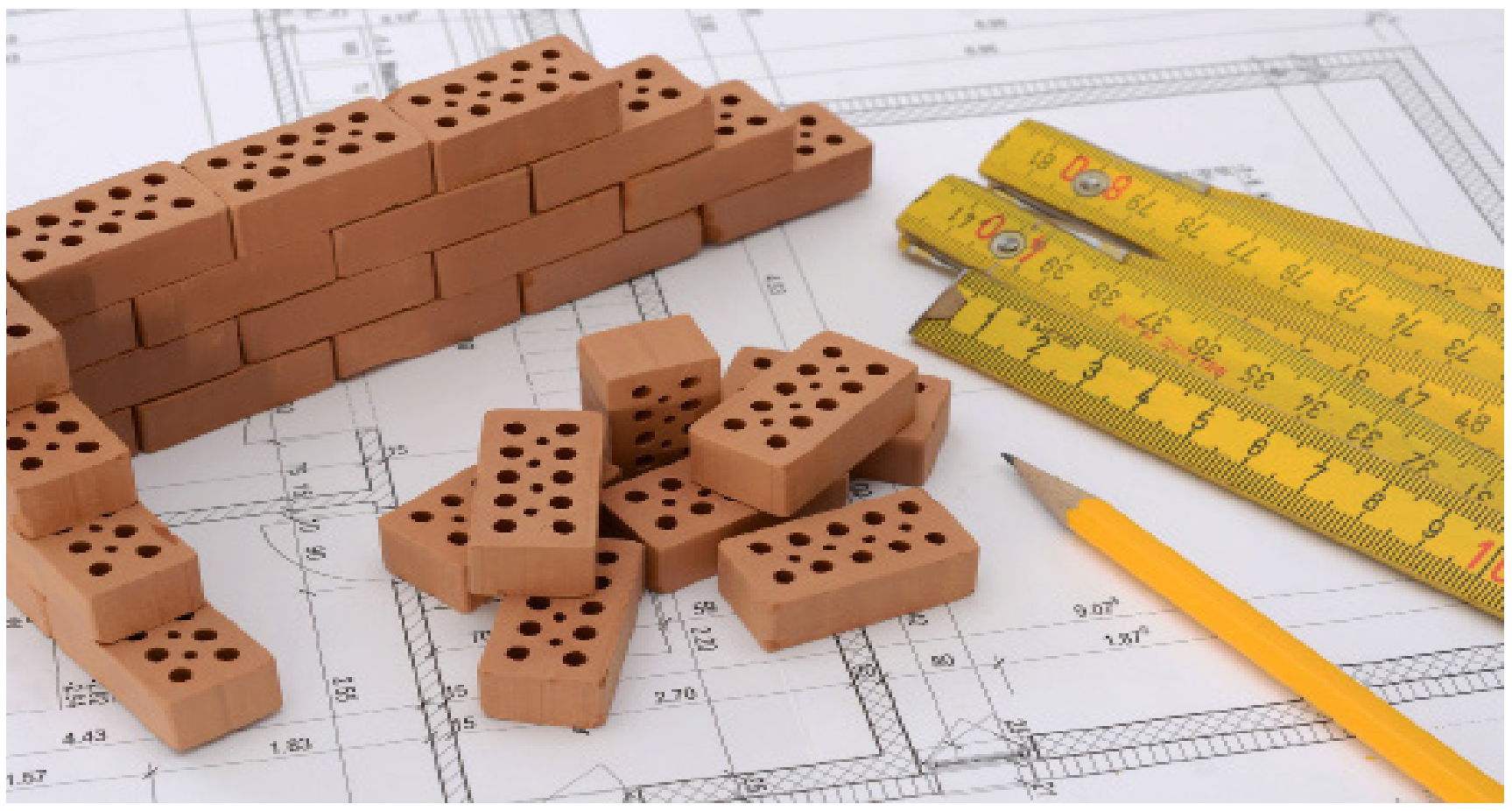

\title{
Prototipo a escala de un modelo de resonancia en edificaciones diseñadas con figuras geométricas
}

\section{Scale prototype of a resonance model in buildings designed with geometric figures}

Andrés Felipe Ruiz-Jiménez

Ingeniero Civil, andresfeliperj@ufps.edu.co,

https://orcid.org/0000-0001-5589-7087, Universidad Francisco de Paula Santander, Cúcuta, Colombia

\section{Fabio Pérez-Márquez}

Magister en Administración de Datacenters,

Fabio.perez@tecnar.edu.co, https://orcid.org/0000-0003-2573-7589,

Docente Investigador, Fundación Tecnológica Antonio de Arevalo -

Unitecnar, Cartagena de Indias, Colombia.
Ender José Barrientos-Monsalve

Arquitecto, enderjosebamo@ufps.edu.co,

https://orcid.org/0000-0001-6673-0223, Universidad Francisco de Paula Santander. Cúcuta, Colombia 


\section{Resumen}

La resonancia como fenómeno físico se presenta en la mayoría de las diversas ramas de la ingeniería como un objeto de estudio, en la ingeniería civil, por ejemplo, este efecto se ve mayormente en sismos y vientos y más específicamente en Colombia lo observamos especialmente en temblores de tierra debido a la localización geográfica del país, por esta razón, en este artículo se realizará un prototipo a escala de un modelo de resonancia en una edificación diseñada con figura geométrica para analizar el por qué se produce este tipo de fenómeno en estas estructuras, evaluar los diferentes movimientos sísmicos que se presentan y determinar como estos generan grandes consecuencias en el diseño estructural de la misma.

Palabras clave:Resonancia, Sismos, Estructuras, Ingeniería, Prototipo, Figura Geométrica.

\section{Abstract}

Resonance as a physical phenomenon is presented in most of the various branches of engineering as a study object, in civil engineering, for example, this effect is seen mainly in earthquakes and winds and more specifically in Colombia we observe it especially in tremors of land due to the geographical location of the country, for this reason, in this article a scale prototype of a resonance model will be made in a building designed with a geometric figure to analyze why this type of phenomenon occurs in these structures, evaluate the different seismic movements that are presented and determine how these generate great consequences in its structural design.

Keywords: Resonance, Earthquakes, Structures, Engineering, Prototype, Geometric Figure. 
Introducción

Las edificaciones están expuestas riesgos de carácter sísmico por diversas causas, que las hacen vulnerables ante estos posibles eventos. Una de estas causas es está relacionada con la resistencia y rigidez de una la estructura determinada, para la cual es fundamenatal el conocimiento de los índices de vulnerabilidad, que permita la adecuación y definición de planes de contingenciavque garantizando la seguridad de los habitantes, en especial si se trata de construcciones antiguas [1-2].

Cada estructura diseñada a partir de figuras geométricas cuenta con un periodo natural (o frecuencia natural) inherente a sí misma. Al entrar la estructura en un estado de excitación externa de vibración (Por ejemplo: un movimiento sísmico, la fuerza del viento, mesa vibradora, un motor sobre una estructura que lo soporte, etc.), este generalmente hace que la estructura adopte un movimiento oscilatorio con una frecuencia determinada [3]. En la ingeniería civil este efecto se ve mayormente en sismos y vientos, y siendo un poco más específicos en Colombia lo observamos especialmente en sismos, a causa de la localización geográfica del país [4].

Asimismo, cuando el sismo excita el suelo, que es la base de las estructuras que están empotradas en él (viviendas, edificios, puentes, etc.) lo somete a una aceleración variable en un movimiento oscilatorio (o de vaivén) tridimensional con una frecuencia variable, y este movimiento es transmitido por medio de la cimentación de las estructuras a los demás elementos estructurales de estas mismas, dando como resultado que toda la estructura adopte una frecuencia de vibración [5].
Cada estructura cuenta con una frecuencia natural inherente, si esta frecuencia que el sismo le impone a la estructura coincide o se acerca a la frecuencia natural de la misma se dará el fenómeno de resonancia, generando unos desplazamientos exagerados en la estructura [6]; por ello, este fenómeno de resonancia es sumamente perjudicial, ya que exige demasiada ductilidad a la estructura, mucha más que la que esta puede dar, con lo que la estructura, mucha más que la que esta puede proveer, con lo que la estructura, naturalmente, buscará la forma de liberar la energía de un modo generalmente destructivo [5].

La resonancia como fenómeno físico se presenta como un objeto de estudio de gran importancia dentro de las diversas ramas de la ingeniería. Se han realizado investigaciones del proyecto teniendo en cuenta los movimientos sísmicos a los cuales ha estado sometido, no sólo en el territorio colombiano sino en el mundo entero. En el marco de este estudio surge la necesidad de evaluar diferentes casos de estudios y analizar la historia de las catástrofes y sus efectos en el sistema territorial, y resulta estratégico trabajar en los diferentes aspectos donde se encuentran impactos por este fenómeno natural [7].

La importancia de esta investigación se origina en el estudio de los efectos y comportamientos de las estructuras, mediante una simulación de modelo físico que sirva como base para desarrollos futuros sobre el fenómeno de la resonancia, y la elaboración de unas maquetas donde se pretende demostrar cómo esta manifestación natural afecta a las edificaciones diseñadas con figuras geométricas.

En el tema de las construcciones estructurales, como el de las edificaciones diseñadas con este tipo de figura, es importante tener en 


\section{0}

cuenta el fenómeno de resonancia desde la fase de diseño, garantizando la protección de la estructura antes las continuas vibraciones que se producen por el movimiento natural del suelo, evitando daños parciales o totales tanto a la estructura como al de los seres vivos [8]; es por ello que la presente investigación tiene como propósito analizar los diferentes prototipos a escala de edificaciones estructurales diseñadas a base de figuras geométricas que están expuestas a movimientos sísmicos.

\section{Antecedentes}

El estudio adelantado por [8] tuvo como objetivo diseñary desarrollar una plataforma de bajo coste que permita enseñar a los alumnos de Ingeniería que estudian asignaturas relacionadas con la materia de Resistencia de materiales y Cálculo de Estructuras los efectos que producen los terremotos, por medio de una metodología: investigación explicativa. Tuvo los siguientes resultados: La efectividad de la mesa de vibraciones propuesta se ha verificado mediante simulaciones numéricas, de tres formas distintas, en las herramientas: Solidworks, y en Matlab (Simulink y con las ecuaciones dinamicas directamente). En estas simulaciones se ha calculado la posición, velocidad, aceleración de la mesa de vibraciones, donde se colocará la maqueta de la estructura a estudiar. En los resultados se pueden apreciar los valores de posición velocidad y aceleración con un perfil de movimiento determinado para el motor del eje $y$, donde se puede apreciar que se toman los valores adecuados para los resultados que se necesitan en este tipo de simulaciones.

Así mismo, se obtiene la fuerza que tendrá la mesa en el eje y, con el ángulo de giro del motor. Con este valor, se puede comparar la reacción que se ha obtenido de esta forma con la calculada mediante las ecuaciones de dinámica. Así no solo se valida el resultado del par, sino que se puede afirmar que el resultado obtenido para las reacciones " $x$ " $e$ "y" es el correcto.

Asimismo [9] tuvo como objetivo abordar el diseño de una mesa vibratoria para movimientos dinámicos controlados en una dirección, para ensayar modelos de estructuras de uno o más grados de libertad, para distintas frecuencias y amplitudes de la solicitación. Con una metodología Investigación Explicativa. Esta tuvo como resultados que mediante el siguiente proyecto se logró entregar un sistema de control capaz de realizar ensayos controlados para distintas frecuencias y amplitudes de oscilación. Dentro de los sistemas de la Mesa Vibratoria podemos concluir que el más relevante es el sistema de control, el cuál fija las principales características del movimiento de la mesa y, por consiguiente, de él depende la obtención de buenos resultados.

En este sistema fue en el que se invirtió mayor cantidad de recursos en relación a tiempo y costos. Asimismo, se entrega una herramienta de estudio y análisis que permite realizar ensayos de modelos de estructuras a escala frente a solicitaciones dinámicas. La Mesa Vibratoria puede realizar ensayos utilizando datos de registros sísmicos, siempre y cuando se respeten sus limitaciones y se entregue la información en el formato adecuado. Al ser una herramienta portable, la Mesa Vibratoria puede trabajar en cualquier lugar ya sea en un aula de clases, un taller o en casa.

De la misma manera [5] en su estudio demostró los posibles efectos que generan los movimientos oscilatorios mediante un modelo físico, el cual permite conocer el movimiento y comportamiento de las estructuras cuando se presentan sismos con diferentes frecuencias. Se utilizò una metodología explicativa. Y tuvo como resultados que los ensayos realizados en el funcionamiento de la mesa vibratoria 
(llustración10), se logró la determinación de las frecuencias en las que cada edificación pierde su estabilidad y entra en el fenómeno de resonancia. La edificación que posee una altura de $0,90 \mathrm{~m}$ y un área de $0,0625 \mathrm{~m} 2$ entró en resonancia al generarse una frecuencia en el sistema de $5,3 \mathrm{~Hz}$; mientras que la estructura que presenta menor altura $(0,45 \mathrm{~m})$ entró en este fenómeno al registrarse $8,7 \mathrm{~Hz}$, es decir, que mediante el ensayo se pudo apreciar que la estructura alta entra en resonancia cuando las longitudes de ondas son de periodo largo, mientras que la estructura más pequeña entra en resonancia cuando estas longitudes de ondas son de periodo corto.

A su vez, se implementaron dispositivos de control para evitar el movimiento de las estructuras, y se logró de manera positiva reducir las respuestas vibratorias del sistema, los aisladores tuvieron un mejor desempeño en el edificio de mayor altura ya que este exigía más por su complejidad en cuanto a masa y altura y por su rápida entrada al fenómeno de la resonancia, en cuanto a la estructura con menor dimensión tuvo una respuesta acorde con lo pretendido por los dispositivos de control activo.

Se concluye que los sistemas aislantes son más efectivos que los sistemas disipadores de energía, ya que los disipadores de energía necesitan un mayor mantenimiento, además a largo plazo afecta negativamente a la estructura generando deformaciones residuales permanentes en ella, por otro lado los sistemas aislantes disminuyen drásticamente los desplazamiento en la estructura causados por un evento sísmico, de esta manera puede proteger y mantener a la edificación operativa durante y después de un sismo severo.

\section{Bases teóricas}

Vibraciones de estructuras. Todas las estructuras que poseen masa y elasticidad son capaces de vibrar. Estas vibraciones pueden ser excitadas por fuentes tales como motores, compresores, vientos, terremotos, etc. Si la frecuencia de estas fuentes de vibración coincide con una de sus frecuencias naturales de vibración, la estructura entra en resonancia y su amplitud de vibración puede alcanzar magnitudes lo suficientemente grandes para dañar o incluso destruirla. La mayoría de los terremotos son el resultado del movimiento rápido a lo largo del plano de fallas dentro de la corteza terrestre. Este movimiento súbito de la falla libera una gran cantidad de energía que viaja a través de la tierra en forma de ondas sísmicas. Las ondas sísmicas viajan grandes distancias antes de perder la mayor parte de su energía [10].

El movimiento de la tierra en el sitio que se encuentra un edificio es muy complicado. No es una onda armónica simple sino una superposición de muchas ondas de frecuencias y amplitudes diferentes. Las características de un terremoto que tienen gran importancia para los edificios son: su duración, su amplitud (de desplazamiento, de velocidad y de aceleración) y su espectro de frecuencia. El movimiento de respuesta del edificio al terremoto es también muy complejo, comienza a vibrar (régimen transitorio) en una manera compleja, en la misma mezcla de frecuencia que tiene el terremoto. Después de un período muy corto, el movimiento se centra alrededor de una las frecuencias naturales de vibración del edificio [5].

En los últimos años los investigadores en el área de la mecánica estructural han ido incrementando las investigaciones en disipadores de energía, aisladores de energía, péndulos contrarestadores de ondas sísmicas, etc. con la finalidad que los edificios sean diseñados acorde con los requerimientos mínimos de fuerza lateral de los códigos de diseño. Recientes sismos han mostrado que los edificios diseñados y construidos de acuerdo a los códigos más recientes 


\section{2}

proveen una buena respuesta, pero el costo de reparación de daños y el tiempo necesario para implementar estas reparaciones son más grandes que las anticipadas [11].

Según [12] tres técnicas innovadoras han sido propuestas para usarse individualmente o en combinación, con la finalidad de mejorar el desempeñosísmico de los edificios: aislamiento sísmico, dispositivos suplementarios de disipación de energía y control estructural activo o híbrido. En la actualidad, dicho sistema constructivo se usa con mucha frecuencia en la práctica y se considera un campo abierto en la investigación sísmica, representando el presente trabajo un aporte importante en la actualización de los métodos de cálculo de edificaciones con dispositivos pasivos de disipación de energía.

Resonancia mecánica. Todo cuerpo tiene una o varias, frecuencias características que "depende mucho de la elasticidad del objeto y/o sistema o de la forma que esté presente. Cuando un sistema es excitado a una de sus frecuencias características, su vibración es la máxima posible. El fenómeno de la resonancia se genera cuando la frecuencia angular de la fuerza externa coincide con la frecuencia natural de oscilación del sistema, con un aumento en la amplitud" [13].

Movimientos periódicos. Un cuerpo que tiene un movimiento periódico es caracterizado por una posición de equilibrio estable. "Cuando se aleja de la posición en la que se encontraba y se suelta, entra en acción una fuerza o torca para volverlo nuevamente a su estado de equilibrio. Sin embargo, en el momento en que se encuentra en equilibrio, ya ha adquirido energía cinética que le permite continuar con el movimiento hasta que se detiene del otro lado, donde nuevamente será impulsado hacia la posición de equilibrio" [5].
Estructura de una edificación vertical. Mientras más compleja es la estructura, más difícil resulta predecir su comportamiento sísmico. Por ello, se recomienda que la estructuración sea lo más simple y limpia posible, de forma tal que el análisis sísmico se acerque lo más posible a la estructura real. "Además se debe evitar que los elementos no estructurales distorsionen la distribución de fuerzas considerada, porque se generarán fuerzas en elementos que no fueron diseñados para esas condiciones" [6].

En un sentido algo amplio, se puede pensar en estructura como un dispositivo proyectado para soportar cargas; donde estas, son sistemas de fuerzas que se ejercen como consecuencia de la función que desempeña la estructura. "En las estructuras de edificación, la finalidad principal de esta es la de soportar aquellas cargas gravitacionales, aunque se tiene presente que pueden presentarse otras cargas de forma ambiental como viento o acciones térmicas" [14].

Simplicidad y simetría. Según estudios realizados a través de los años, se ha podido determinar y demostrar que las estructuras simples son las que mejor comportamiento tienen en el momento de presentarse un sismo. El ejecutar un modelo para realizar el análisis estructural de un pórtico sobre una placa, puede ser sencillo y los resultados obtenidos pueden reflejar con alguna precisión el comportamiento real que presenta la estructura; sin embargo, cuando las estructuras son tan complejas, se presentan dificultades en el modelo a realizar, por lo que no va a tener una buena precisión o asegurar la similitud del modelo y el comportamiento real que debería tener la estructura.

"La simetría de la estructura en dos direcciones es deseable debido a que la falta de simetría produce efectos torsionales que son difíciles de evaluar y pueden ser muy destructivos. Las fuerzas de los sismos se pueden idealizar 
si se trabaja en el centro de masas de cada piso, mientras que las fuerzas que absorben los elementos estarán ubicadas en el centro de rigidez; si no existe coincidencia entre estos puntos 49 (centro de masas y centro de rigidez), el movimiento sísmico se trasladará y adicionalmente se generará una torsión, la que incrementará los esfuerzos debidos al sismo, sobrepasándose los esfuerzos resistentes, como se puede observar en la siguiente ilustración" [12].

Resultados

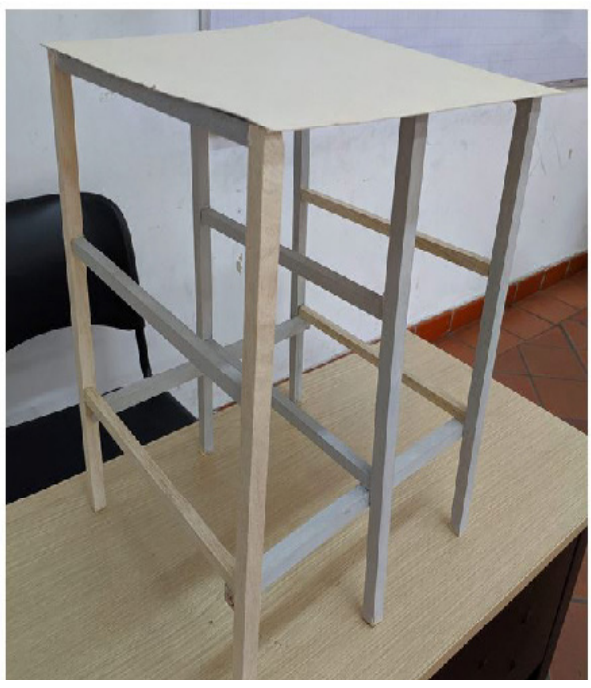

Figura 1. Modelo a escala de la estructura

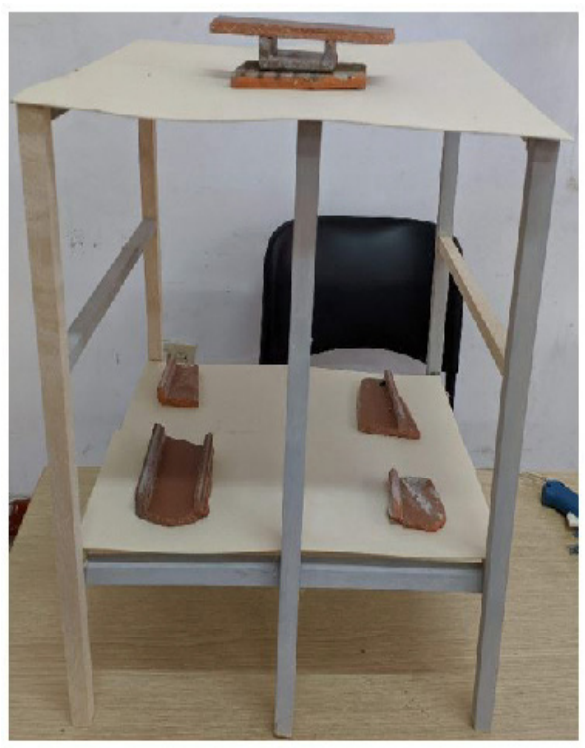

Figura 2. Cargas actuantes en la estructura
Una vez instaladas las estructuras, se realizaron variaciones en la implementación de materiales, porque los materiales en madera y las placas en cartón no cumplían con el propósito del proyecto debido a que son materiales ligeramente rígidos para la altura de diseño sin embargo cuando se unieron los extremos de la estructura cumplió con la flexibilidad que se pretendía, esto debido a la variación de masa que hay entre los materiales conforme a la escala del proyecto.

\section{Conclusiones}

Con base al funcionamiento del prototipo se pudo concluir que, la estructura que presenta una mayor masa necesitó de una baja frecuencia para poder entrar en resonancia, por otro lado, la misma edificación con la misma altura y menor masa requirió de una mayor velocidad ejercida para que la frecuencia aumentara y coincidiera con la frecuencia de la estructura con el fin de conseguir sus movimientos resonantes.

Una de las soluciones para los problemas de inseguridad estructural e incomodidad ante las vibraciones en edificios altos, es el manejo de aisladores y disipadores para el control pasivo de vibraciones, sin la utilización de energía externa mediante el cual se logra reducir de forma significativa la magnitud de las vibraciones en las estructuras. Es una alternativa que se presenta como una de las formas más eficaces y de bajo costo para el mejoramiento de la seguridad de estructuras existentes.

Se recomienda que antes de diseñar el modelo se debe realizar un estudio detallado de aspectos tales como la elección de la geometría esbozada, las cantidades de material presupuestadas con sus respectivos costos y la selección de los materiales de acuerdo a sus características y lo que se pretende con el proyecto, con el fin de efectuar 


\section{4}

la menor cantidad posible de reformas al diseño inicial, ahorrando tiempo en la consecución del proyecto.

\section{Referencias}

[1] A. Rodríguez Lizcano, R. Centeno Lara, "Aplicación de las tic en el análisis y percepción del nivel de vulnerabilidad de edificaciones residenciales de la ciudad de Cúcuta," Revista Ingenio, vol. 11, no. 1, pp. 19-26, diciembre 2016.

[2] J.F. Márquez-Peñaranda, "Análisis de la vulnerabilidad sísmica de los Edificios Fundadores y Aulas Sur de la U.F.P.S", Respuestas, vol. 8, no. 1, pp. 38-54, jun. 2016

[3] C. Vargas, Antecedentes generales de la aislación y disipación de energía sísmica, 2015. [En línea]. Disponible en: https://antisismos. blogspot.com/2015/06/111-antecedentesgenerales-de-la.html

[4] F. Vidal Sánchez, Los terremotos y sus causas. Granada, España: Instituto Andaluz de Geofísica y Prevención de Desastres Sísmicos, 2009

[5] F. Cárdenas Hernández y C. Cepeda Isidro, Prototipo a escala de un modelo de resonancia en edificaciones para el laboratorio de Ingenieria civil de la Universidad Católica de Colombia, trabajo de grado, Universidad Católica de Colombia, Bogotá, 2016

[6] D. Moreno. I. YamiN y J.C Reyes, Estudio Experimental de Aisladores Sísmicos Tipo Péndulo de Fricción en Modelos a Escala Reducida. Universidad de los Andes. Colombia, 2006

[7] M.Y. González Sandoval, Estudio de la interacción suelo-estructura en el rango elástico e inelástico de una zapata aislada mediante modelos físicos a escala y mediante elementos finitos, trabajo de grado, Escuela Colombiana de Ingeniería Julio Garavito, 2015

[8] D.B. Suarez Mamani, Respuesta en resonancia de una estructura tipo marco excitada armónicamente en la base, trabajo de grado, Universidad Nacional de San Antonio Abad del Cusco, 2017

[9] Á. Artero Real, J.L. Blanco Claraco, A. Giménez Fernández, J. López Martínez y J.L. Torres Moreno, Simulador sísmico de bajo coste para uso en prácticas en docencia, en Actas de las XXXIX Jornadas de Automática, Badajoz, 5-7 de Septiembre de 2018

[10] F.J. Oyarce Anguita, "Diseño y construcción de una mesa vibratoria unidireccional portable, para ensayos de modelos a escala de estructuras para distintas condiciones de frecuencia y amplitud de la solicitación", trabajo de grado, Universidad Austral de Chile, Valdivia, Chile, 2015

[11] M.Y. Molestina Luzuriaga, Diseño fabricación y ensayo de una mesa de excitación dinámica para modelos estructurales a escala, trabajo de grado, Universidad San Francisco de Quito, 2014

[12] J.A. Badillo Lascano, Comparación del comportamiento a la resonancia entre estructuras con muros de corte duales y estructuras con pórticos resistentes a momento para un suelo típico de Quito, trabajo de grado, Pontificia Universidad Católica del Ecuador, 2015

[13] G. Villareal Castro, Las estructuras. Bellavista: Universidad Nacional del Callao, 2011

[14] J.P. Torres, Resonancia Tema 1.15. México D.F.: Departamento de Astronomía Universidad de Guanajuato, 2009. [En línea]. Disponible en:http://www.astro.ugto.mx/ papaqui/ ondasyfluidos/Tema_1.15-Resonancia.pdf 